\title{
Least-Squares Based and Gradient Based Iterative Parameter Estimation Algorithms for a Class of Linear-in-Parameters Multiple-Input Single-Output Output Error Systems
}

\author{
Cheng Wang, Tao Tang, and Dewang Chen \\ State Key Laboratory of Rail Traffic Control and Safety, Beijing Jiaotong University, Beijing 100044, China \\ Correspondence should be addressed to Cheng Wang; artiefly@gmail.com
}

Received 27 May 2014; Accepted 21 July 2014; Published 13 August 2014

Academic Editor: Giuseppe Marino

Copyright (C) 2014 Cheng Wang et al. This is an open access article distributed under the Creative Commons Attribution License, which permits unrestricted use, distribution, and reproduction in any medium, provided the original work is properly cited.

The identification of a class of linear-in-parameters multiple-input single-output systems is considered. By using the iterative search, a least-squares based iterative algorithm and a gradient based iterative algorithm are proposed. A nonlinear example is used to verify the effectiveness of the algorithms, and the simulation results show that the least-squares based iterative algorithm can produce more accurate parameter estimates than the gradient based iterative algorithm.

\section{Introduction}

Parameter estimation plays an important role in adaptive forecasting [1], system modeling [2-6], system control [7-9], and adaptive control [10-15]. For decades, many identification methods have been developed [16-20], for example, the bias compensation based least-squares methods [21-23] and the iterative identification methods [24-26]. These methods can be used for identifying linear systems and nonlinear systems. In the literature, Ding presented a decomposition based fast least-squares algorithm for output error systems [27]. Recursive algorithms and iterative algorithms are two types of parameter estimation algorithms. The recursive algorithms use the data as it becomes available [28], whereas the iterative algorithms tend to exploit the advantage of processing a complete batch of available data, which can provide highly accurate parameter estimation. Iterative methods can also be used for solving matrix equations [29-31]. In the literature, Ding proposed a two-stage least-squares based iterative parameter estimation algorithm for CARARMA systems using the decomposition technique [32].

As a basic class of multivariable systems, multiple-input single-output (MISO) systems have lots of applications in industrial processes. Several works on MISO system identification have been reported [33]. For example, in order to improve the convergence rate, Liu et al. developed a stochastic gradient algorithm for MISO systems using the multi-innovation theory [34]. The least-squares methods can also be found in the literature.

Recently, Wang and Tang studied the identification algorithms for a class of linear-in-parameters single-input singleoutput (SISO) systems with colored noises using the recursive least-squares method [35]. In this work, we extend these results from SISO systems into a class of linear-in-parameters MISO systems with the colored noises shown in Figure 1 $[36,37]$. Consider

$$
y(t)=\sum_{j=1}^{r} \frac{\boldsymbol{\vartheta}_{j}^{\mathrm{T}} \boldsymbol{\eta}_{j}\left(u_{j}(t)\right)}{A_{j}(z)}+D(z) v(t),
$$

where $y(t) \in \mathbb{R}$ is the system output, $\left\{u_{j}(t) \in \mathbb{R}, j=\right.$ $1,2, \ldots, r\}$ are the system inputs, and $v(t) \in \mathbb{R}$ is the stochastic white noise with zero mean. $A_{j}(z)$ and $D(z)$ are polynomials, of known orders $\left(n_{j}, n_{d}\right)$, in the unit backward shift operator $z^{-1}$, and defined by 


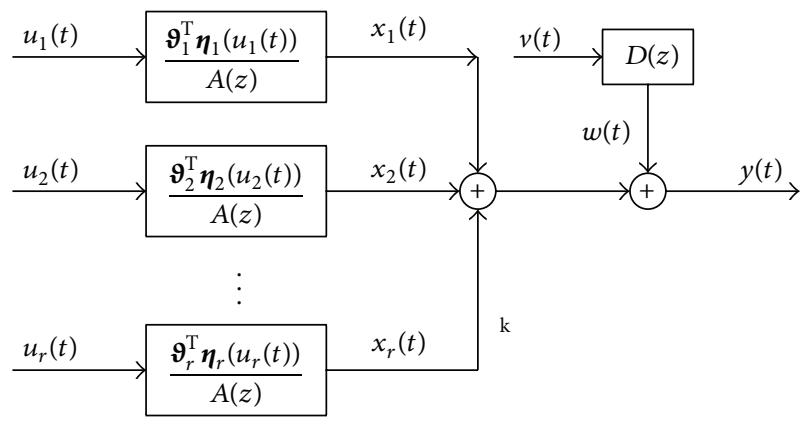

Figure 1: The linear-in-parameters multiple-input single-output output error moving average systems.

$$
\begin{gathered}
A_{j}(z):=1+a_{j 1} z^{-1}+a_{j 2} z^{-2}+\cdots+a_{j n_{j}} z^{-n_{j}} \in \mathbb{R}, \\
D(z):=1+d_{1} z^{-1}+d_{2} z^{-2}+\cdots+d_{n_{d}} z^{-n_{d}} \in \mathbb{R},
\end{gathered}
$$

$a_{j i}, d_{i}$, and $\boldsymbol{\vartheta}_{j}^{\mathrm{T}}$ are the unknown parameters to be estimated. The superscript $\mathrm{T}$ denotes the matrix/vector transpose. It is worth noting that the models in (1) include but are not limited to linear MISO systems; that is, when $\boldsymbol{\vartheta}_{j}$ and $\boldsymbol{\eta}_{j}\left(u_{j}(t)\right)$ are defined by

$$
\begin{gathered}
\boldsymbol{\vartheta}_{j}:=\left[b_{j 1}, b_{j 2}, \ldots, b_{j n_{j}}\right]^{\mathrm{T}} \in \mathbb{R}^{n_{j}}, \\
\boldsymbol{\eta}_{j}\left(u_{j}(t)\right):=\left[u_{j}(t), u_{j}(t-1), \ldots, u_{j}\left(t-n_{j}\right)\right]^{\mathrm{T}} \in \mathbb{R}^{n_{j}},
\end{gathered}
$$

system (1) denotes an MISO output error moving average system. When $\boldsymbol{\eta}_{j}\left(u_{j}(t)\right)$ is a nonlinear function of $u_{j}(t)$, for example,

$$
\begin{aligned}
\boldsymbol{\eta}_{j} & \left(u_{j}(t)\right) \\
& :=\left[\sin \left(u_{j}(t-1)\right), u_{j}^{3}(t), \ldots, \sqrt{2} \tan \left(u_{j}\left(t-n_{j}\right)\right)\right]^{\mathrm{T}} \in \mathbb{R}^{n_{j}},
\end{aligned}
$$

system (1) denotes a nonlinear MISO system.

On the basis of the iterative algorithms for linear-inparameters SISO systems $[37,38]$, this paper develops the least-squares based and gradient based iterative identification algorithms to improve the parameter estimation accuracy for a class of linear-in-parameters MISO output error moving average systems. Compared with the gradient based iterative algorithm, the least-squares based iterative algorithm can provide more accurate parameter estimates.

The remainder of this paper is organized as follows. Section 2 introduces the identification model. Section 3 derives the least-squares based iterative algorithm. Section 4 proposes a gradient based iterative algorithm. Section 5 presents an illustrative example to show the effectiveness of the algorithms. Finally, concluding remarks are offered in Section 6.

\section{The Identification Model}

Let us define some symbols. The symbol $\mathbf{I}_{n}$ denotes an identity matrix of order $n ; \mathbf{1}_{n}$ denotes an $n$-dimensional column vector whose elements are $1 ; \lambda_{\max }[\mathbf{X}]$ and $\mathbf{X}^{-1}$ represent the maximum eigenvalue and the inverse of the square matrix $\mathbf{X}$.

To further develop new identification algorithms for estimating the parameter vector $\boldsymbol{\vartheta}_{j}$ and the parameters of $A_{j}(z)$ and $D(z)$ by utilizing the input-output measured data $\left\{u_{j}(t), y(t): t=1,2, \ldots\right\}$, we derive an identification model for system (1). Without loss of generality, assume that $u_{j}(t)=$ $0, y(t)=0$, and $v(t)=0$ for $t \leq 0$.

Define the intermediate variables as follows:

$$
\begin{aligned}
x_{j}(t) & :=\frac{\boldsymbol{\vartheta}_{j}^{\mathrm{T}} \boldsymbol{\eta}_{j}\left(u_{j}(t)\right)}{A_{j}(z)} \\
& =-\sum_{i=1}^{n_{a}} a_{j i} x_{j}(t-i)+\boldsymbol{\vartheta}_{j}^{\mathrm{T}} \boldsymbol{\eta}_{j}\left(u_{j}(t)\right), \\
w(t) & :=D(z) v(t) .
\end{aligned}
$$

Define the parameter vectors as follows:

$$
\begin{gathered}
\boldsymbol{\theta}:=\left[\begin{array}{c}
\boldsymbol{\theta}_{\mathrm{s}} \\
\boldsymbol{\theta}_{\mathrm{n}}
\end{array}\right] \in \mathbb{R}^{n_{0}+n_{d}}, \\
\boldsymbol{\theta}_{\mathrm{s}}:=\left[\boldsymbol{\theta}_{1}^{\mathrm{T}}, \boldsymbol{\theta}_{2,}^{\mathrm{T}}, \ldots, \boldsymbol{\theta}_{r}^{\mathrm{T}}\right]^{\mathrm{T}} \in \mathbb{R}^{n_{0}}, \quad n_{0}:=2 \sum_{j=1}^{r} n_{j}, \\
\boldsymbol{\theta}_{\mathrm{n}}:=\left[d_{1}, d_{2}, \ldots, d_{n_{d}}\right]^{\mathrm{T}} \in \mathbb{R}^{n_{d}}, \\
\boldsymbol{\theta}_{j}:=\left[a_{j 1}, a_{j 2}, \ldots, a_{j n_{j}}, \boldsymbol{\vartheta}_{j}^{\mathrm{T}}\right]^{\mathrm{T}} \in \mathbb{R}^{2 n_{j}},
\end{gathered}
$$

and define the information vectors as follows:

$$
\begin{gathered}
\boldsymbol{\varphi}(t):=\left[\begin{array}{c}
\boldsymbol{\varphi}_{\mathrm{s}}(t) \\
\boldsymbol{\varphi}_{\mathrm{n}}(t)
\end{array}\right] \in \mathbb{R}^{n_{0}+n_{d}}, \\
\boldsymbol{\varphi}_{\mathrm{s}}(t):=\left[\boldsymbol{\varphi}_{1}^{\mathrm{T}}(t), \boldsymbol{\varphi}_{2}^{\mathrm{T}}(t), \ldots, \boldsymbol{\varphi}_{r}^{\mathrm{T}}(t)\right]^{\mathrm{T}} \in \mathbb{R}^{n_{0}}, \\
n_{0}=2 \sum_{j=1}^{r} n_{j}, \\
\boldsymbol{\varphi}_{\mathrm{n}}(t):=\left[-v(t-1),-v(t-2), \ldots,-v\left(t-n_{d}\right)\right]^{\mathrm{T}} \in \mathbb{R}^{n_{d}}, \\
\boldsymbol{\varphi}_{j}(t):=\left[-x_{j}(t-1),-x_{j}(t-2), \ldots,-x_{j}\left(t-n_{j}\right),\right. \\
\left.\boldsymbol{\eta}_{j}^{\mathrm{T}}\left(u_{j}(t)\right)\right]^{\mathrm{T}} \in \mathbb{R}^{2 n_{j}} .
\end{gathered}
$$

Then we can express (5) as

$$
\begin{gathered}
x_{j}(t)=\boldsymbol{\varphi}_{J}^{\mathrm{T}}(t) \boldsymbol{\theta}_{j}, \\
w(t)=\boldsymbol{\varphi}_{\mathrm{n}}^{\mathrm{T}}(t) \boldsymbol{\theta}_{\mathrm{n}}+v(t),
\end{gathered}
$$

and system (1) can be rewritten as

$$
\begin{aligned}
y(t) & =\sum_{j=1}^{r} x_{j}(t)+w(t) \\
& =\boldsymbol{\varphi}_{\mathrm{s}}^{\mathrm{T}}(t) \boldsymbol{\theta}_{\mathrm{s}}+\boldsymbol{\varphi}_{\mathrm{n}}^{\mathrm{T}}(t) \boldsymbol{\theta}_{\mathrm{n}}+v(t) \\
& =\boldsymbol{\varphi}^{\mathrm{T}}(t) \boldsymbol{\theta}+v(t) .
\end{aligned}
$$


Equation (9) is the identification model of system (1), and parameter vector $\boldsymbol{\theta}$ contains all the parameters of the system.

\section{The Least-Squares Based Iterative Algorithm}

Consider the newest $p$ data from $t-p+1$ to $t$ and define the quadratic criterion function as follows:

$$
J(\boldsymbol{\theta}):=\sum_{i=0}^{p-1}\left[y(t-i)-\boldsymbol{\varphi}^{\mathrm{T}}(t-i) \boldsymbol{\theta}\right]^{2} .
$$

By minimizing $J(\boldsymbol{\theta})$ and letting the derivative of $J(\boldsymbol{\theta})$ with respect to $\boldsymbol{\theta}$ be zero, we can obtain the least-squares estimate of $\boldsymbol{\theta}$ as

$$
\widehat{\boldsymbol{\theta}}(t)=\left[\sum_{i=0}^{p-1} \boldsymbol{\varphi}(t-i) \boldsymbol{\varphi}^{\mathrm{T}}(t-i)\right]^{-1}\left[\sum_{i=0}^{p-1} \boldsymbol{\varphi}(t-i) y(t-i)\right] .
$$

The above estimate $\widehat{\boldsymbol{\theta}}(t)$ is impossible to implement due to the unknown noise-free outputs $x_{j}(t-i)$ and unmeasurable noise items $v(t-i)$ in $\varphi(t)$. Here, the difficulties are solved by using the iterative identification technique [38]: let $k=1,2, \ldots$ be the iterative variable, and let $\widehat{\boldsymbol{\theta}}_{j, k}(t)$ and $\widehat{\boldsymbol{\theta}}_{k}(t)$ be the iterative estimates of $\boldsymbol{\theta}_{j}$ and $\boldsymbol{\theta}$ at iteration $k$, replace the unknown items $x_{j}(t-i)$ and $v(t-i)$ with their iterative estimates $\widehat{x}_{j, k}(t-i)$ and $\widehat{v}_{k}(t-i)$ at iteration $k$, and define the estimated vectors as follows:

$$
\begin{gathered}
\widehat{\boldsymbol{\varphi}}_{k}(t):=\left[\widehat{\boldsymbol{\varphi}}_{\mathrm{s}, k}^{\mathrm{T}}(t), \widehat{\boldsymbol{\varphi}}_{\mathrm{n}, k}^{\mathrm{T}}(t)\right]^{\mathrm{T}} \in \mathbb{R}^{n_{0}+n_{d}}, \\
\widehat{\boldsymbol{\varphi}}_{\mathrm{s}, k}(t):=\left[\widehat{\boldsymbol{\varphi}}_{1, k}^{\mathrm{T}}(t), \widehat{\boldsymbol{\varphi}}_{2, k}^{\mathrm{T}}(t), \ldots, \widehat{\boldsymbol{\varphi}}_{r, k}^{\mathrm{T}}(t)\right]^{\mathrm{T}} \in \mathbb{R}^{n_{0}}, \\
n_{0}=2 \sum_{j=1}^{r} n_{j}, \\
\widehat{\boldsymbol{\varphi}}_{\mathrm{n}, k}(t):=\left[\widehat{v}_{k}(t-1), \widehat{v}_{k}(t-2), \ldots, \widehat{v}_{k}\left(t-n_{d}\right)\right]^{\mathrm{T}} \in \mathbb{R}^{n_{d}}, \\
\widehat{\boldsymbol{\varphi}}_{j, k}(t):=\left[-\widehat{x}_{j, k}(t-1),-\widehat{x}_{j, k}(t-2), \ldots,\right. \\
\left.-\widehat{x}_{j, k}\left(t-n_{j}\right), \boldsymbol{\eta}_{j}^{\mathrm{T}}\left(u_{j}(t)\right)\right]^{\mathrm{T}} \in \mathbb{R}^{2 n_{j}} .
\end{gathered}
$$

Let $\widehat{\boldsymbol{\theta}}_{j, k}(t)$ and $\widehat{\boldsymbol{\theta}}_{k}(t)$ be the estimates of $\boldsymbol{\theta}_{j}$ and $\boldsymbol{\theta}$ at iteration $k$, let $\widehat{x}_{j, k}(t)$ and $\widehat{v}_{k}(t)$ be the estimates of $x_{j}(t)$ and $v(t)$ at iteration $k$. Replacing $\varphi(t-i)$ in (11) with its corresponding estimate $\widehat{\boldsymbol{\varphi}}_{k}(t-i)$, we can obtain the following least-squares based iterative algorithm for MISO systems in (1) (the MISOLSI algorithm for short) into:

$$
\begin{gathered}
\widehat{\boldsymbol{\theta}}(t)=\left[\sum_{i=0}^{p-1} \widehat{\boldsymbol{\varphi}}_{k}(t-i) \widehat{\boldsymbol{\varphi}}_{k}^{\mathrm{T}}(t-i)\right]^{-1}\left[\sum_{i=0}^{p-1} \widehat{\boldsymbol{\varphi}}_{k}(t-i) y(t-i)\right], \\
\widehat{\boldsymbol{\varphi}}_{k}(t)=\left[\widehat{\boldsymbol{\varphi}}_{\mathrm{s}, k}^{\mathrm{T}}(t), \hat{\boldsymbol{\varphi}}_{\mathrm{n}, k}^{\mathrm{T}}(t)\right]^{\mathrm{T}} \\
\widehat{\boldsymbol{\varphi}}_{\mathrm{s}, k}(t)=\left[\widehat{\boldsymbol{\varphi}}_{1, k}^{\mathrm{T}}(t), \widehat{\boldsymbol{\varphi}}_{2, k}^{\mathrm{T}}(t), \ldots, \widehat{\boldsymbol{\varphi}}_{r, k}^{\mathrm{T}}(t)\right]^{\mathrm{T}}
\end{gathered}
$$

$$
\begin{gathered}
\widehat{\boldsymbol{\varphi}}_{j, k}(t)=\left[-\widehat{x}_{j, k}(t-1),-\widehat{x}_{j, k}(t-2), \ldots,-\widehat{x}_{j, k}\left(t-n_{j}\right),\right. \\
\left.\boldsymbol{\eta}_{j}^{\mathrm{T}}\left(u_{j}(t)\right)\right]^{\mathrm{T}}, \\
\widehat{\boldsymbol{\varphi}}_{\mathrm{n}, k}(t)=\left[\widehat{v}_{k}(t-1), \widehat{v}_{k}(t-2), \ldots, \widehat{v}_{k}\left(t-n_{d}\right)\right]^{\mathrm{T}}, \\
\widehat{x}_{j, k}(t-i)=\widehat{\boldsymbol{\varphi}}_{j, k}^{\mathrm{T}}(t-i) \widehat{\boldsymbol{\theta}}_{j, k}(t), \\
\widehat{v}_{k}(t-i)=y(t-i)-\widehat{\boldsymbol{\varphi}}_{k}^{\mathrm{T}}(t-i) \widehat{\boldsymbol{\theta}}_{k}(t), \\
\widehat{\boldsymbol{\theta}}_{k}(t)=\left[\widehat{\boldsymbol{\theta}}_{\mathrm{s}, k}^{\mathrm{T}}(t), \widehat{\boldsymbol{\theta}}_{\mathrm{n}, k}^{\mathrm{T}}(t)\right]^{\mathrm{T}} \\
\widehat{\boldsymbol{\theta}}_{\mathrm{s}, k}(t)=\left[\widehat{\boldsymbol{\theta}}_{1, k}^{\mathrm{T}}(t), \widehat{\boldsymbol{\theta}}_{2, k}^{\mathrm{T}}(t), \ldots, \widehat{\boldsymbol{\theta}}_{r, k}^{\mathrm{T}}(t)\right]^{\mathrm{T}}, \\
\widehat{\boldsymbol{\theta}}_{\mathrm{n}, k}(t)=\left[\widehat{d}_{1, k}(t), \widehat{d}_{2, k}(t), \ldots, \widehat{d}_{n_{d}, k}(t)\right]^{\mathrm{T}}, \\
\widehat{\boldsymbol{\theta}}_{j, k}(t):=\left[\widehat{a}_{j 1, k}(t), \widehat{a}_{j 2, k}(t), \ldots, \widehat{a}_{j n_{j}, k}(t), \widehat{\boldsymbol{\theta}}_{j, k}^{\mathrm{T}}(t)\right]^{\mathrm{T}} .
\end{gathered}
$$

The steps of computing $\widehat{\boldsymbol{\theta}}_{k}(t)$ involved in the algorithm are summarized as follows.

(1) Given $p$, let $t=p$ and collect the input-output data $\left\{u_{j}(i), y(i): i=0,1, \ldots, p-1\right\}$.

(2) Collect the present input-output data $u_{j}(t)$ and $y(t)$.

(3) To initialize, let $k=1, \widehat{x}_{j, 0}(t-i)=$ random number, and $\widehat{v}_{0}(t-i)=$ random number, $i=$ $1,2, \ldots, \max \left[n_{j}, n_{d}\right]$.

(4) Form $\widehat{\boldsymbol{\varphi}}_{\mathrm{s}, k}(t-i)$ using (15) and $\widehat{\boldsymbol{\varphi}}_{\mathrm{n}, k}(t-i)$ using (17), and form $\widehat{\boldsymbol{\varphi}}_{j, k}(t-i)$ using (16) and $\widehat{\boldsymbol{\varphi}}_{k}(t)$ using (14).

(5) Update the parameter estimate $\widehat{\boldsymbol{\theta}}_{k}(t)$ using (13).

(6) Compute $\widehat{x}_{j, k}(t-i)$ using (18) and $\widehat{v}_{k}(t-i)$ using (19).

(7) If $\left\|\widehat{\boldsymbol{\theta}}_{k}(t)-\widehat{\boldsymbol{\theta}}_{k-1}(t)\right\| \leqslant \varepsilon$ (a given small number), obtain the iterative time $k$ and the parameter estimate $\widehat{\boldsymbol{\theta}}_{k}(t)$; let $\widehat{\boldsymbol{\theta}}_{0}(t+1)=\widehat{\boldsymbol{\theta}}_{k}(t)$, increase $t$ by 1 , and go to Step 2; otherwise, increase $k$ by 1 and go to Step 4 .

\section{The Gradient Based Iterative Algorithm}

By minimizing $J(\boldsymbol{\theta})$ through the negative gradient search, we obtain the following recursive relation of computing the estimate of $\boldsymbol{\theta}$ at iteration $k$ :

$$
\begin{aligned}
& \widehat{\boldsymbol{\theta}}_{k}(t)= \widehat{\boldsymbol{\theta}}_{k-1}(t)+\frac{\mu_{k}(t)}{2} \operatorname{grad}\left[J\left(\widehat{\boldsymbol{\theta}}_{k-1}(t)\right)\right] \\
&=\widehat{\boldsymbol{\theta}}_{k-1}(t)+\mu_{k}(t) \sum_{i=0}^{p-1} \boldsymbol{\varphi}(t-i) \\
& \quad \times\left[y(t-i)-\boldsymbol{\varphi}^{\mathrm{T}}(t-i) \widehat{\boldsymbol{\theta}}_{k-1}(t)\right],
\end{aligned}
$$

where $\mu_{k}(t)$ is the step-size or the convergence factor to be given later. The same difficulties arise in that the noise-free 
outputs $x_{j}(t-i)$ in $\varphi_{s}(t)$ and the noise items $v(t-i)$ in $\boldsymbol{\varphi}_{\mathrm{n}}(t)$ of $\boldsymbol{\varphi}(t)$ on the right-hand side of (24) are unknown. Here we apply the same scheme used in the previous section, replacing the unknown vectors with their corresponding iterative estimates. Referring to the method in [38], replacing $\boldsymbol{\varphi}(t-i)$ in $(24)$ with $\widehat{\boldsymbol{\varphi}}_{k}(t-i)$, we can summarize the following gradient based iterative algorithm for MISO systems in (1) (the MISO-GI algorithm for short):

$$
\begin{aligned}
& \widehat{\boldsymbol{\theta}}_{k}(t)=\widehat{\boldsymbol{\theta}}_{k-1}(t)+\mu_{k}(t) \\
& \times \sum_{i=0}^{p-1} \widehat{\boldsymbol{\varphi}}_{k}(t-i)\left[y(t-i)-\widehat{\boldsymbol{\varphi}}_{k}^{\mathrm{T}}(t-i) \widehat{\boldsymbol{\theta}}_{k-1}(t)\right], \\
& \widehat{\boldsymbol{\varphi}}_{k}(t)=\left[\widehat{\boldsymbol{\varphi}}_{\mathrm{s}, k}^{\mathrm{T}}(t), \widehat{\boldsymbol{\varphi}}_{\mathrm{n}, k}^{\mathrm{T}}(t)\right]^{\mathrm{T}}, \\
& \widehat{\boldsymbol{\varphi}}_{s, k}(t)=\left[\widehat{\boldsymbol{\varphi}}_{1, k}^{\mathrm{T}}(t), \widehat{\boldsymbol{\varphi}}_{2, k}^{\mathrm{T}}(t), \ldots, \widehat{\boldsymbol{\varphi}}_{r, k}^{\mathrm{T}}(t)\right]^{\mathrm{T}} \text {, } \\
& \widehat{\boldsymbol{\varphi}}_{j, k}(t)=\left[-\widehat{x}_{j, k}(t-1),-\widehat{x}_{j, k}(t-2), \ldots,-\widehat{x}_{j, k}\left(t-n_{j}\right),\right. \\
& \left.\boldsymbol{\eta}_{j}^{\mathrm{T}}\left(u_{j}(t)\right)\right]^{\mathrm{T}} \\
& \widehat{\boldsymbol{\varphi}}_{\mathrm{n}, k}(t)=\left[\widehat{v}_{k}(t-1), \widehat{v}_{k}(t-2), \ldots, \widehat{v}_{k}\left(t-n_{d}\right)\right]^{\mathrm{T}}, \\
& \widehat{x}_{j, k}(t-i)=\widehat{\boldsymbol{\varphi}}_{j, k}^{\mathrm{T}}(t-i) \widehat{\boldsymbol{\theta}}_{j, k}(t), \\
& \widehat{v}_{k}(t-i)=y(t-i)-\widehat{\boldsymbol{\varphi}}_{k}^{\mathrm{T}}(t-i) \widehat{\boldsymbol{\theta}}_{k}(t) \text {, } \\
& \mu_{k}(t) \leqslant 2 \lambda_{\max }^{-1}\left[\sum_{i=0}^{p-1} \widehat{\boldsymbol{\varphi}}_{k}(t-i) \hat{\boldsymbol{\varphi}}_{k}^{\mathrm{T}}(t-i)\right], \\
& \widehat{\boldsymbol{\theta}}_{k}(t)=\left[\widehat{\boldsymbol{\theta}}_{\mathrm{s}, k}^{\mathrm{T}}(t), \widehat{\boldsymbol{\theta}}_{\mathrm{n}, k}^{\mathrm{T}}(t)\right]^{\mathrm{T}} \text {, } \\
& \widehat{\boldsymbol{\theta}}_{\mathrm{s}, k}(t)=\left[\widehat{\boldsymbol{\theta}}_{1, k}^{\mathrm{T}}(t), \widehat{\boldsymbol{\theta}}_{2, k}^{\mathrm{T}}(t), \ldots, \widehat{\boldsymbol{\theta}}_{r, k}^{\mathrm{T}}(t)\right]^{\mathrm{T}}, \\
& \widehat{\boldsymbol{\theta}}_{\mathrm{n}, k}(t)=\left[\widehat{d}_{1, k}(t), \widehat{d}_{2, k}(t), \ldots, \widehat{d}_{n_{d}, k}(t)\right]^{\mathrm{T}} \text {, } \\
& \widehat{\boldsymbol{\theta}}_{j, k}(t):=\left[\widehat{a}_{j 1, k}(t), \widehat{a}_{j 2, k}(t), \ldots, \widehat{a}_{j n_{j}, k}(t), \widehat{\boldsymbol{\vartheta}}_{j, k}^{\mathrm{T}}(t)\right]^{\mathrm{T}} \text {. }
\end{aligned}
$$

The steps of computing $\widehat{\boldsymbol{\theta}}_{k}(t)$ involved in the algorithm are summarized as follows.

(1) Given $p$, let $t=p$ and collect the input-output data $\left\{u_{j}(i), y(i): i=0,1, \ldots, p-1\right\}$ set $\widehat{\boldsymbol{\theta}}_{0}(t)=\mathbf{1}_{n_{0}} / p_{0}$, $p_{0}=10^{6}$.

(2) Collect the present input-output data $u_{j}(t)$ and $y(t)$.

(3) To initialize, let $k=1, \widehat{x}_{j, 0}(t-i)=1 / p_{0}$, and $\widehat{v}_{0}(t-i)=$ $1 / p_{0}, i=1,2, \ldots, \max \left[n_{j}, n_{d}\right]$.

(4) Form $\widehat{\boldsymbol{\varphi}}_{\mathrm{s}, k}(t-i)$ using (27) and $\widehat{\boldsymbol{\varphi}}_{\mathrm{n}, k}(t-i)$ using (29), and form $\widehat{\boldsymbol{\varphi}}_{j, k}(t-i)$ using (28).

(5) Choose an appropriate step-size $\mu_{k}(t)$ using (32) and update the parameter estimate $\widehat{\boldsymbol{\theta}}_{k}(t)$ using (25).

(6) Compute $\widehat{x}_{j, k}(t-i)$ using (30) and $\widehat{v}_{k}(t-i)$ using (31).
(7) If $\left\|\widehat{\boldsymbol{\theta}}_{k}(t)-\widehat{\boldsymbol{\theta}}_{k-1}(t)\right\| \leqslant \varepsilon$ (a given small number), obtain the iterative time $k$ and the parameter estimate $\widehat{\boldsymbol{\theta}}_{k}(t)$; let $\widehat{\boldsymbol{\theta}}_{0}(t+1)=\widehat{\boldsymbol{\theta}}_{k}(t)$, increase $t$ by 1 , and go to Step 2; otherwise, increase $k$ by 1 and go to Step 4 .

\section{Example}

Consider the following nonlinear multiple-input singleoutput simulation system:

$$
\begin{gathered}
y(t)=\frac{\boldsymbol{\vartheta}_{1}^{\mathrm{T}} \boldsymbol{\eta}_{1}\left(u_{1}(t)\right)}{A_{1}(z)}+\frac{\boldsymbol{\vartheta}_{2}^{\mathrm{T}} \boldsymbol{\eta}_{2}\left(u_{2}(t)\right)}{A_{2}(z)}+D(z) v(t) \\
A_{1}(z)=1+a_{1} z^{-1}=1-0.38 z^{-1}, \\
A_{2}(z)=1+a_{2} z^{-1}=1-0.44 z^{-1}, \\
D(z)=1+d_{1} z^{-1}=1+0.69 z^{-1}, \\
\boldsymbol{\eta}_{1}\left(u_{1}(t)\right)=u_{1}(t-1) \\
\boldsymbol{\eta}_{2}\left(u_{2}(t)\right)=u_{2}^{2}(t-1) \\
\boldsymbol{\vartheta}_{1}=b_{1}=1.48 \\
\boldsymbol{\vartheta}_{2}=b_{2}=1.58 \\
\boldsymbol{\theta}=\left[a_{1}, b_{1}, a_{2}, b_{2}, d_{1}\right]^{\mathrm{T}}=[-0.38,1.48,-0.44,1.58,0.69]^{\mathrm{T}} .
\end{gathered}
$$

Here, the inputs $\left\{u_{1}(t)\right\}$ and $\left\{u_{2}(t)\right\}$ are taken as uncorrelated persistent excitation signal sequences with zero means and unit variances and $\{v(t)\}$ as a white noise sequence with zero mean.

Using $t=p=1000$ data and applying the MISO-GI algorithm in (25)-(32) and the MISO-LSI algorithm in (13)(19) to estimate the parameters of this nonlinear system, the parameter estimates of each algorithm and their errors with noise variance $\sigma^{2}=0.50^{2}$ are shown in Table 1 ; the parameter estimation errors $\delta:=\left\|\widehat{\boldsymbol{\theta}}_{k}(t)-\boldsymbol{\theta}\right\| /\|\boldsymbol{\theta}\|$ versus $k$ of each algorithm are illustrated in Figure 2. We also investigate the performance of two algorithms under a relatively high noise level with noise variance $\sigma^{2}=1.00^{2}$, and the corresponding simulation results are illustrated in Table 2 and Figure 3.

From the simulation results in Tables 1 and 2 and Figures 2 and 3, we can draw the following conclusions.

(i) The parameter estimation errors are getting smaller as the iterative variable $k$ increases.

(ii) Both algorithms can produce highly accurate parameter estimates under different noise variances.

(iii) The MISO-LSI algorithm converges faster than the MISO-GI algorithm does; however, due to the use of a batch of data, the MISO-LSI algorithm involves many matrix computations, resulting in the high computational complexity. One possible solution for reducing the computational load of the MISO-LSI algorithm with large $p$ is using the decomposition technique [27], which is widely adopted in the leastsquares based iterative algorithms. 
TABLE 1: The MISO-GI and MISO-LSI estimates and errors $\left(\sigma^{2}=0.50^{2}\right)$.

\begin{tabular}{|c|c|c|c|c|c|c|c|}
\hline Algorithm & $k$ & $a_{1}$ & $b_{1}$ & $a_{2}$ & $b_{2}$ & $d_{1}$ & $\delta(\%)$ \\
\hline \multirow{6}{*}{ MISO-GI } & 1 & 0.01462 & 1.27390 & 0.09679 & 0.89451 & -0.07066 & 52.82221 \\
\hline & 2 & -0.23411 & 1.31836 & -0.13385 & 1.15839 & 0.62397 & 24.24129 \\
\hline & 5 & -0.33873 & 1.45005 & -0.48982 & 1.43125 & 0.77055 & 7.82671 \\
\hline & 8 & -0.35503 & 1.47142 & -0.47912 & 1.46594 & 0.73773 & 5.64270 \\
\hline & 10 & -0.35781 & 1.47633 & -0.47364 & 1.48458 & 0.72409 & 4.65183 \\
\hline & 20 & -0.36024 & 1.48089 & -0.45659 & 1.54144 & 0.69498 & 1.99007 \\
\hline \multirow{6}{*}{ MISO-LSI } & 1 & 0.03460 & 1.41912 & -0.00027 & 2.25122 & -0.02164 & 49.09578 \\
\hline & 2 & -0.37155 & 1.46913 & -0.44592 & 1.58467 & 0.41478 & 11.75374 \\
\hline & 5 & -0.36086 & 1.48239 & -0.44390 & 1.58413 & 0.68642 & 0.87072 \\
\hline & 8 & -0.36082 & 1.48242 & -0.44387 & 1.58420 & 0.68670 & 0.87077 \\
\hline & 10 & -0.36082 & 1.48242 & -0.44387 & 1.58420 & 0.68670 & 0.87077 \\
\hline & 20 & -0.36082 & 1.48242 & -0.44387 & 1.58420 & 0.68670 & 0.87077 \\
\hline \multicolumn{2}{|c|}{ True values } & -0.38000 & 1.48000 & -0.44000 & 1.58000 & 0.69000 & \\
\hline
\end{tabular}

TABLE 2: The MISO-GI and MISO-LSI estimates and errors $\left(\sigma^{2}=1.00^{2}\right)$.

\begin{tabular}{|c|c|c|c|c|c|c|c|}
\hline Algorithm & $k$ & $a_{1}$ & $b_{1}$ & $a_{2}$ & $b_{2}$ & $d_{1}$ & $\delta(\%)$ \\
\hline \multirow{6}{*}{ MISO-GI } & 1 & 0.02747 & 1.25718 & 0.09726 & 0.89934 & -0.06054 & 52.75453 \\
\hline & 2 & -0.13980 & 1.28713 & -0.06373 & 1.08128 & 0.58629 & 30.02651 \\
\hline & 5 & -0.31243 & 1.44812 & -0.49633 & 1.42666 & 0.73252 & 7.87070 \\
\hline & 8 & -0.33623 & 1.47067 & -0.48609 & 1.46212 & 0.68840 & 5.72438 \\
\hline & 10 & -0.33927 & 1.47574 & -0.48043 & 1.48105 & 0.68211 & 4.89225 \\
\hline & 20 & -0.34152 & 1.48130 & -0.46229 & 1.54025 & 0.68242 & 2.56420 \\
\hline \multirow{6}{*}{ MISO-LSI } & 1 & 0.04885 & 1.40155 & 0.00051 & 2.26283 & -0.00903 & 49.33764 \\
\hline & 2 & -0.36198 & 1.46011 & -0.45714 & 1.57034 & 0.44031 & 10.74006 \\
\hline & 5 & -0.34220 & 1.48370 & -0.44808 & 1.58781 & 0.68523 & 1.70082 \\
\hline & 8 & -0.34214 & 1.48373 & -0.44804 & 1.58792 & 0.68545 & 1.70303 \\
\hline & 10 & -0.34214 & 1.48373 & -0.44804 & 1.58792 & 0.68545 & 1.70303 \\
\hline & 20 & -0.34214 & 1.48373 & -0.44804 & 1.58792 & 0.68545 & 1.70303 \\
\hline True v & & -0.38000 & 1.48000 & -0.44000 & 1.58000 & 0.69000 & \\
\hline
\end{tabular}

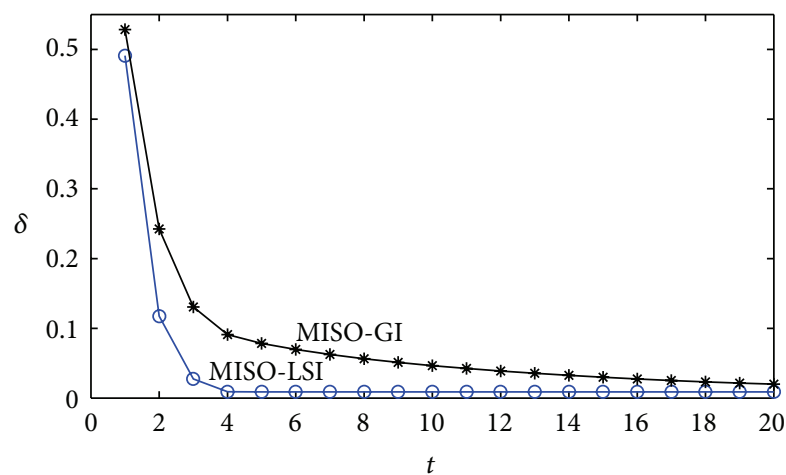

Figure 2: The parameter estimation errors $\delta$ versus $t\left(\sigma^{2}=0.50^{2}\right)$.

\section{Conclusions}

In this work, we have presented two iterative identification algorithms, a least-squares based iterative algorithm and a gradient based iterative algorithm, for a class of linearin-parameters multiple-input single-output output error

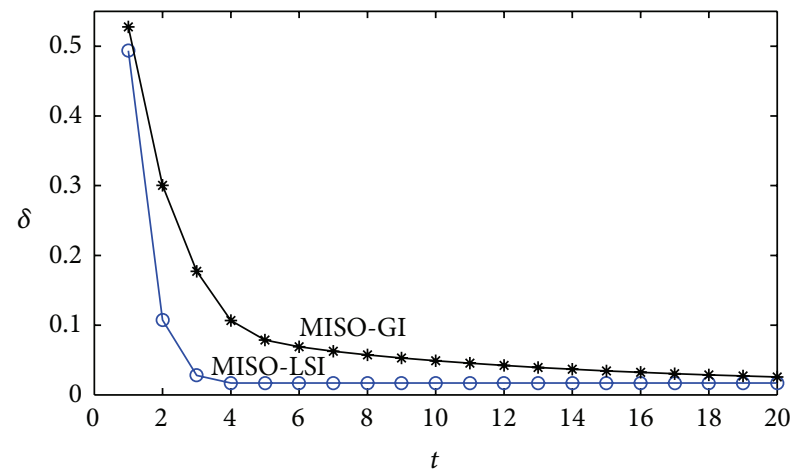

Figure 3: The parameter estimation errors $\delta$ versus $t\left(\sigma^{2}=1.00^{2}\right)$.

moving average systems. The illustrative example shows that both algorithms can provide more accurate parameter estimates. The proposed methods can be extended to study the identification problems of linear multivariable systems [39, 40] or multirate or nonuniformly sampled systems [41, 42]. The methods in this paper can combine the multi-innovation 
identification methods [43-50], the iterative identification methods [51, 52], and other identification methods [53-56] to present new identification algorithms for nonlinear systems [57-59] and can also be applied in other fields [60-67].

\section{Conflict of Interests}

The authors declare that there is no conflict of interests regarding the publication of this paper.

\section{Acknowledgments}

This work was supported by the "Beijing Laboratory For Mass Transit" Program (I13H100010) and the Project of Beijing Municipal Science and Technology (D141100000814002).

\section{References}

[1] P. J. Smith, K. J. Beven, A. H. Weerts, and D. Leedal, "Adaptive correction of deterministic models to produce probabilistic forecasts," Hydrology and Earth System Sciences, vol. 16, no. 8, pp. 2783-2799, 2012.

[2] F. Ding, "Hierarchical estimation algorithms for multivariable systems using measurement information," Information Sciences, vol. 277, pp. 396-405, 2014.

[3] Y. Hu, "Iterative and recursive least squares estimation algorithms for moving average systems," Simulation Modelling Practice and Theory, vol. 34, pp. 12-19, 2013.

[4] F. Ding, "State filtering and parameter identification for state space systems with scarce measurements," Signal Processing, vol. 104, pp. 369-380, 2014.

[5] Y. B. Hu, B. L. Liu, Q. Zhou, and C. Yang, "Recursive extended least squares parameter estimation for Wiener nonlinear systems with moving average noises," Circuits, Systems, and Signal Processing, vol. 33, no. 2, pp. 655-664, 2014.

[6] F. Ding, P. X. Liu, and H. Yang, "Parameter identification and intersample output estimation for dual-rate systems," IEEE Transactions on Systems, Man, and Cybernetics A:Systems and Humans, vol. 38, no. 4, pp. 966-975, 2008.

[7] X. L. Luan, P. Shi, and F. Liu, "Stabilization of networked control systems with random delays," IEEE Transactions on Industrial Electronics, vol. 58, no. 9, pp. 4323-4330, 2011.

[8] X. Luan, S. Zhao, and F. Liu, " $H_{\infty}$ control for discrete-time Markov jump systems with uncertain transition probabilities," IEEE Transactions on Automatic Control, vol. 58, no. 6, pp. 15661572, 2013.

[9] P. Shi, X. Luan, and F. Liu, " $H_{\infty}$ filtering for discrete-time systems with stochastic incomplete measurement and mixed delays," IEEE Transactions on Industrial Electronics, vol. 59, no. 6, pp. 2732-2739, 2012.

[10] Y. Shi and H. Fang, "Kalman filter-based identification for systems with randomly missing measurements in a network environment," International Journal of Control, vol. 83, no. 3, pp. 538-551, 2010.

[11] Y. Shi and B. Yu, "Output feedback stabilization of networked control systems with random delays modeled by Markov chains," IEEE Transactions on Automatic Control, vol. 54, no. 7, pp. 1668-1674, 2009.

[12] Y. Shi and B. Yu, "Robust mixed H-2/H-infinity control of networked control systems with random time delays in both forward and backward communication links," Automatica, vol. 47, no. 4, pp. 754-760, 2011.

[13] J. Herrera, A. Ibeas, S. Alcántara, and M. de la Sen, "Multimodel-based techniques for the identification and adaptive control of delayed multi-input multi-output systems," IET Control Theory \& Applications, vol. 5, no. 1, pp. 188-202, 2011.

[14] F. Ding and T. Chen, "Least squares based self-tuning control of dual-rate systems," International Journal of Adaptive Control and Signal Processing, vol. 18, no. 8, pp. 697-714, 2004.

[15] J. Zhang, F. Ding, and Y. Shi, "Self-tuning control based on multi-innovation stochastic gradient parameter estimation," Systems and Control Letters, vol. 58, no. 1, pp. 69-75, 2009.

[16] D. Wang, F. Ding, and Y. Chu, "Data filtering based recursive least squares algorithm for Hammerstein systems using the keyterm separation principle," Information Sciences, vol. 222, pp. 203-212, 2013.

[17] J. H. Li, "Parameter estimation for Hammerstein CARARMA systems based on the Newton iteration," Applied Mathematics Letters, vol. 26, no. 1, pp. 91-96, 2013.

[18] Y. Liu, F. Ding, and Y. Shi, "An efficient hierarchical identification method for general dual-rate sampled-data systems," Automatica, vol. 50, no. 3, pp. 962-970, 2014.

[19] J. Vörös, "Parameter identification of Wiener systems with multisegment piecewise-linear nonlinearities," Systems and Control Letters, vol. 56, no. 2, pp. 99-105, 2007.

[20] Y. Zhang and G. M. Cui, "Bias compensation methods for stochastic systems with colored noise," Applied Mathematical Modelling, vol. 35, no. 4, pp. 1709-1716, 2011.

[21] Y. Zhang, "Unbiased identification of a class of multi-input single-output systems with correlated disturbances using bias compensation methods," Mathematical and Computer Modelling, vol. 53, no. 9-10, pp. 1810-1819, 2011.

[22] T. Söderström, "Comparing some classes of bias-compensating least squares methods," Automatica. A Journal of IFAC, the International Federation of Automatic Control, vol. 49, no. 3, pp. 840-845, 2013.

[23] J. Ding and F. Ding, "Bias compensation-based parameter estimation for output error moving average systems," International Journal of Adaptive Control and Signal Processing, vol. 25, no. 12, pp. 1100-1111, 2011.

[24] F. Ding, Y. J. Liu, and B. Bao, "Gradient based and least squares based iterative estimation algorithms for multi-input multioutput systems," Proceedings of the Institution of Mechanical Engineers I: Journal of Systems and Control Engineering, vol. 226, no. 1, pp. 43-55, 2012.

[25] D. Q. Wang, "Least squares-based recursive and iterative estimation for output error moving average systems using data filtering," IET Control Theory and Applications, vol. 5, no. 14, pp. 1648-1657, 2011.

[26] F. Ding, X. G. Liu, and J. Chu, "Gradient-based and leastsquares-based iterative algorithms for Hammerstein systems using the hierarchical identification principle," IET Control Theory \& Applications, vol. 7, no. 2, pp. 176-184, 2013.

[27] F. Ding, "Decomposition based fast least squares algorithm for output error systems," Signal Processing, vol. 93, no. 5, pp. 12351242, 2013.

[28] W. Xiong, W. Fan, and R. Ding, "Least-squares parameter estimation algorithm for a class of input nonlinear systems," Journal of Applied Mathematics, vol. 2012, Article ID 684074, 14 pages, 2012. 
[29] M. Hajarian, “The generalized QMRCGSTAB algorithm for solving Sylvester-transpose matrix equations," Applied Mathematics Letters, vol. 26, no. 10, pp. 1013-1017, 2013.

[30] M. Dehghan and M. Hajarian, "On the generalized reflexive and anti-reflexive solutions to a system of matrix equations," Linear Algebra and its Applications, vol. 437, no. 11, pp. 2793-2812, 2012.

[31] L. Xie, Y. Liu, and H. Yang, "Gradient based and least squares based iterative algorithms for matrix equations $A X B+C X^{T} D$ = F," Applied Mathematics and Computation, vol. 217, no. 5, pp. 2191-2199, 2010.

[32] F. Ding, "Two-stage least squares based iterative estimation algorithm for CARARMA system modeling," Applied Mathematical Modelling, vol. 37, no. 7, pp. 4798-4808, 2013.

[33] F. Ding, T. Chen, and L. Qiu, "Bias compensation based recursive least-squares identification algorithm for MISO systems," IEEE Transactions on Circuits and Systems II: Express Briefs, vol. 53, no. 5, pp. 349-353, 2006.

[34] Y. Liu, Y. Xiao, and X. Zhao, "Multi-innovation stochastic gradient algorithm for multiple-input single-output systems using the auxiliary model," Applied Mathematics and Computation, vol. 215, no. 4, pp. 1477-1483, 2009.

[35] C. Wang and T. Tang, "Recursive least squares estimation algorithm applied to a class of linear-in-parameters output error moving average systems," Applied Mathematics Letters. An International Journal of Rapid Publication, vol. 29, pp. 36-41, 2014.

[36] F. Ding, System Identification-New Theory and Methods, Science Press, Beijing, China, 2013.

[37] C. Wang and T. Tang, "Several gradient-based iterative estimation algorithms for a class of nonlinear systems using the filtering technique," Nonlinear Dynamics, vol. 77, no. 3, pp. 769780, 2014.

[38] F. Ding, X. M. Liu, H. B. Chen, and G. Y. Yao, "Hierarchical gradient based and hierarchical least squares based iterative parameter identification for CARARMA systems," Signal Processing, vol. 97, pp. 31-39, 2014.

[39] Y. J. Liu, J. Sheng, and R. F. Ding, "Convergence of stochastic gradient estimation algorithm for multivariable ARX-like systems," Computers and Mathematics with Applications, vol. 59, no. 8, pp. 2615-2627, 2010.

[40] F. Ding, "Coupled-least-squares identification for multivariable systems," IET Control Theory \& Applications, vol. 7, no. 1, pp. 68-79, 2013.

[41] J. Ding, F. Ding, X. P. Liu, and G. Liu, "Hierarchical least squares identification for linear SISO systems with dual-rate sampleddata," IEEE Transactions on Automatic Control, vol. 56, no. 11, pp. 2677-2683, 2011.

[42] L. Xie, H. Yang, and B. Huang, "FIR model identification of multirate processes with random delays using EM algorithm," AICHE Journal, vol. 59, no. 11, pp. 4124-4132, 2013.

[43] F. Ding and T. Chen, "Performance analysis of multi-innovation gradient type identification methods," Automatica. A Journal of IFAC, the International Federation of Automatic Control, vol. 43, no. 1, pp. 1-14, 2007.

[44] F. Ding, P. X. Liu, and G. Liu, "Multiinnovation least-squares identification for system modeling," IEEE Transactions on Systems, Man, and Cybernetics, Part B: Cybernetics, vol. 40, no. 3, pp. 767-778, 2010.

[45] F. Ding, X. P. Liu, and G. Liu, "Auxiliary model based multiinnovation extended stochastic gradient parameter estimation with colored measurement noises," Signal Processing, vol. 89, no. 10, pp. 1883-1890, 2009.
[46] F. Ding, "Several multi-innovation identification methods," Digital Signal Processing, vol. 20, no. 4, pp. 1027-1039, 2010.

[47] L. Xie, Y. J. Liu, H. Z. Yang, and F. Ding, "Modelling and identification for non-uniformly periodically sampled-data systems," IET Control Theory \&Applications, vol. 4, no. 5, pp. 784-794, 2010.

[48] Y. J. Liu, L. Yu, and F. Ding, "Multi-innovation extended stochastic gradient algorithm and its performance analysis," Circuits, Systems, and Signal Processing, vol. 29, no. 4, pp. 649667, 2010.

[49] F. Ding, G. Liu, and X. P. Liu, "Parameter estimation with scarce measurements," Automatica. A Journal of IFAC, the International Federation of Automatic Control, vol. 47, no. 8, pp. 1646-1655, 2011.

[50] F. Ding, "Hierarchical multi-innovation stochastic gradient algorithm for Hammerstein nonlinear system modeling," Applied Mathematical Modelling, vol. 37, no. 4, pp. 1694-1704, 2013.

[51] X. Liu and J. Lu, "Least squares based iterative identification for a class of multirate systems," Automatica, vol. 46, no. 3, pp. 549$554,2010$.

[52] L. Xie and H. Z. Yang, "Gradient-based iterative identification for nonuniform sampling output error systems," Journal of Vibration and Control, vol. 17, no. 3, pp. 471-478, 2011.

[53] J. Ding, C. X. Fan, and J. X. Lin, "Auxiliary model based parameter estimation for dual-rate output error systems with colored noise," Applied Mathematical Modelling, vol. 37, no. 6, pp. 4051-4058, 2013.

[54] J. Ding and J. X. Lin, "Modified subspace identification for periodically non-uniformly sampled systems by using the lifting technique," Systems and Signal Processing, vol. 33, no. 5, pp. 1439-1449, 2014.

[55] L. Xie, Y. Zhao, D. Aziz et al., "Soft sensors for online steam quality measurements of OTSGs," Journal of Process Control, vol. 23, no. 7, pp. 990-1000, 2013.

[56] L. Xie and H. Z. Yang, "Interactive parameter estimation for output error moving average systems," Transactions of the Institute of Measurement and Control, vol. 35, no. 1, pp. 34-43, 2013.

[57] D. Wang and F. Ding, "Least squares based and gradient based iterative identification for Wiener nonlinear systems," Signal Processing, vol. 91, no. 5, pp. 1182-1189, 2011.

[58] D. Wang and F. Ding, "Hierarchical least squares estimation algorithm for hammerstein-wiener systems," IEEE Signal Processing Letters, vol. 19, no. 12, pp. 825-828, 2012.

[59] F. Ding, X. P. Liu, and G. Liu, "Identification methods for Hammerstein nonlinear systems," Digital Signal Processing: A Review Journal, vol. 21, no. 2, pp. 215-238, 2011.

[60] D. Q. Zhu and M. Kong, "Adaptive fault-tolerant control of non-linear systems: an improved CMAC-based fault learning approach," International Journal of Control, vol. 80, no. 10, pp. 1576-1594, 2007.

[61] D. Q. Zhu, J. Liu, and S. X. Yang, "Particle swarm optimization approach to thruster fault-tolerant control of unmanned underwater vehicles," International Journal of Robotics and Automation, vol. 26, no. 3, pp. 426-432, 2011.

[62] D. Zhu, Q. Liu, and Z. Hu, "Fault-tolerant control algorithm of the manned submarine with multi-thruster based on quantumbehaved particle swarm optimisation," International Journal of Control, vol. 84, no. 11, pp. 1817-1829, 2011. 
[63] D. Q. Zhu, Y. Zhao, and M. Z. Yan, "A bio-inspired neurodynamics based back stepping path-following control of an AUV with ocean current," International Journal of Robotics and Automation, vol. 27, no. 3, pp. 298-307, 2012.

[64] Q. Liu, D. Zhu, and S. X. Yang, "Unmanned underwater vehicles fault identification and fault-tolerant control method based on FCA-CMAC neural networks, applied on an actuated vehicle," Journal of Intelligent and Robotic Systems, vol. 66, no. 4, pp. 463475, 2012.

[65] D. Zhu, H. Huang, and S. X. Yang, "Dynamic task assignment and path planning of multi-auv system based on an improved self-organizing map and velocity synthesis method in 3D underwater workspace," IEEE Transactions on Cybernetics, vol. 43, no. 2, pp. 504-514, 2013.

[66] D. Q. Zhu, B. Sun, and S. X. Yang, "The bio-inspired neural network based hybrid sliding-mode tracking control for openframe underwater vehicles," Engineering Applications of Artificial Intelligence, vol. 26, no. 4, pp. 2260-2269, 2013.

[67] B. Sun, D. Q. Zhu, and S. X. Yang, "A bio-inspired filtered backstepping cascaded tracking control of $7000 \mathrm{~m}$ manned submarine vehicle," IEEE Transactions on Industrial Electronics, vol. 61, no. 7, pp. 3682-3692, 2013. 


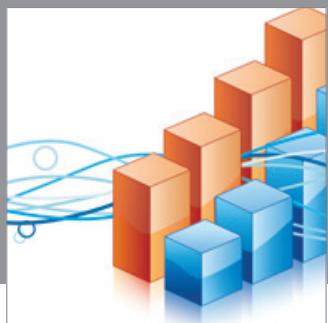

Advances in

Operations Research

mansans

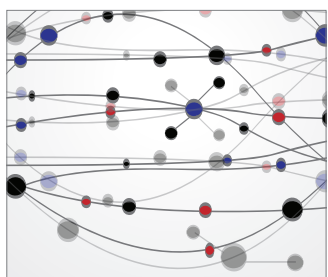

The Scientific World Journal
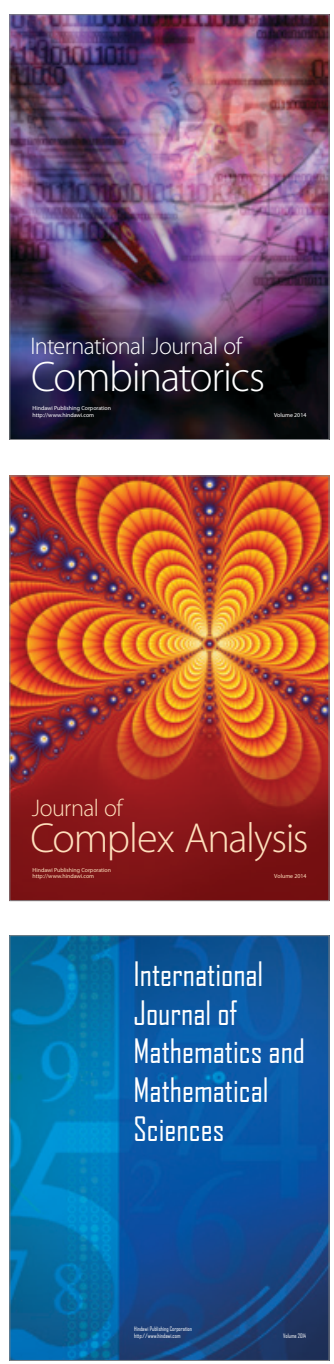
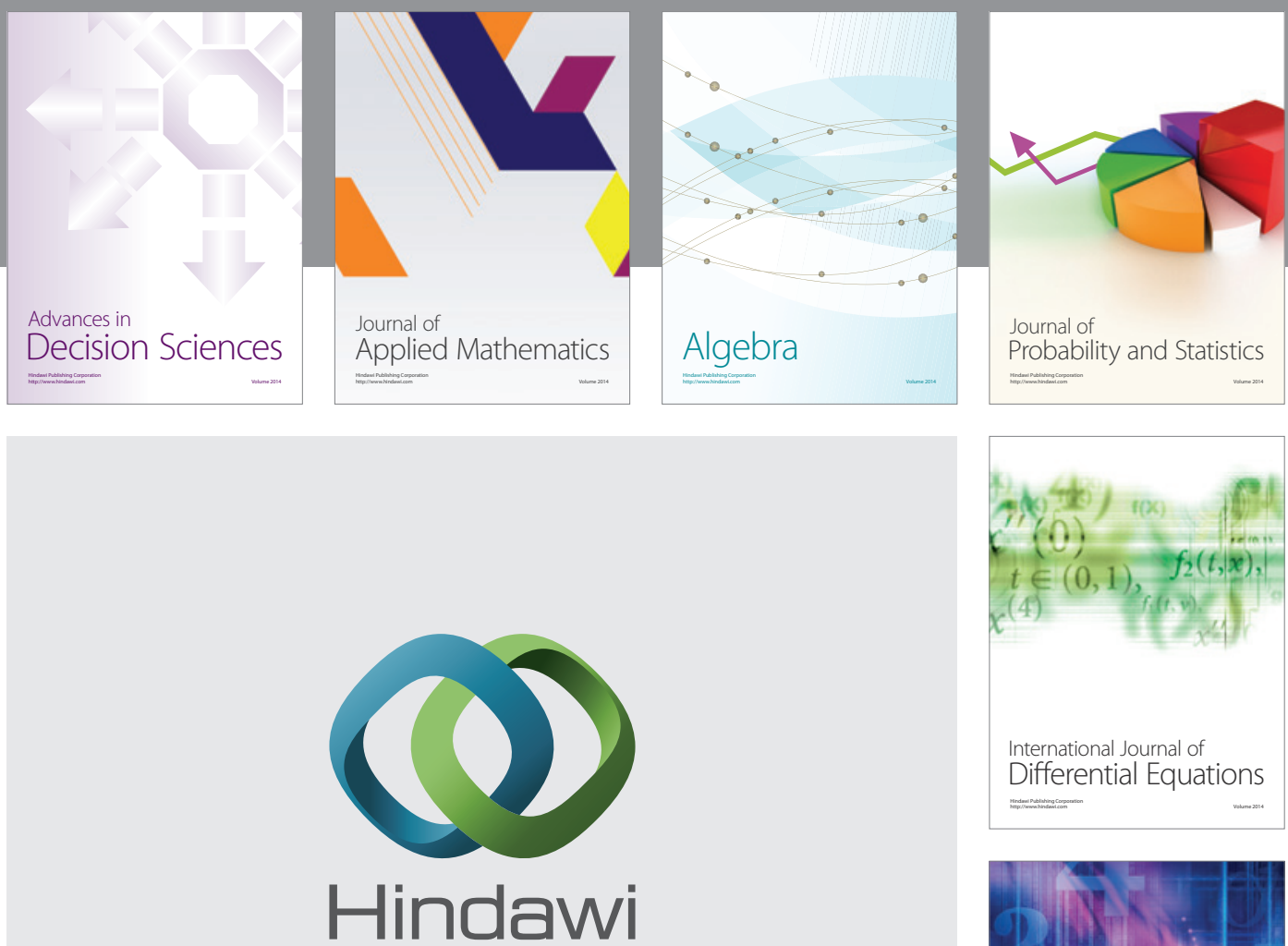

Submit your manuscripts at http://www.hindawi.com
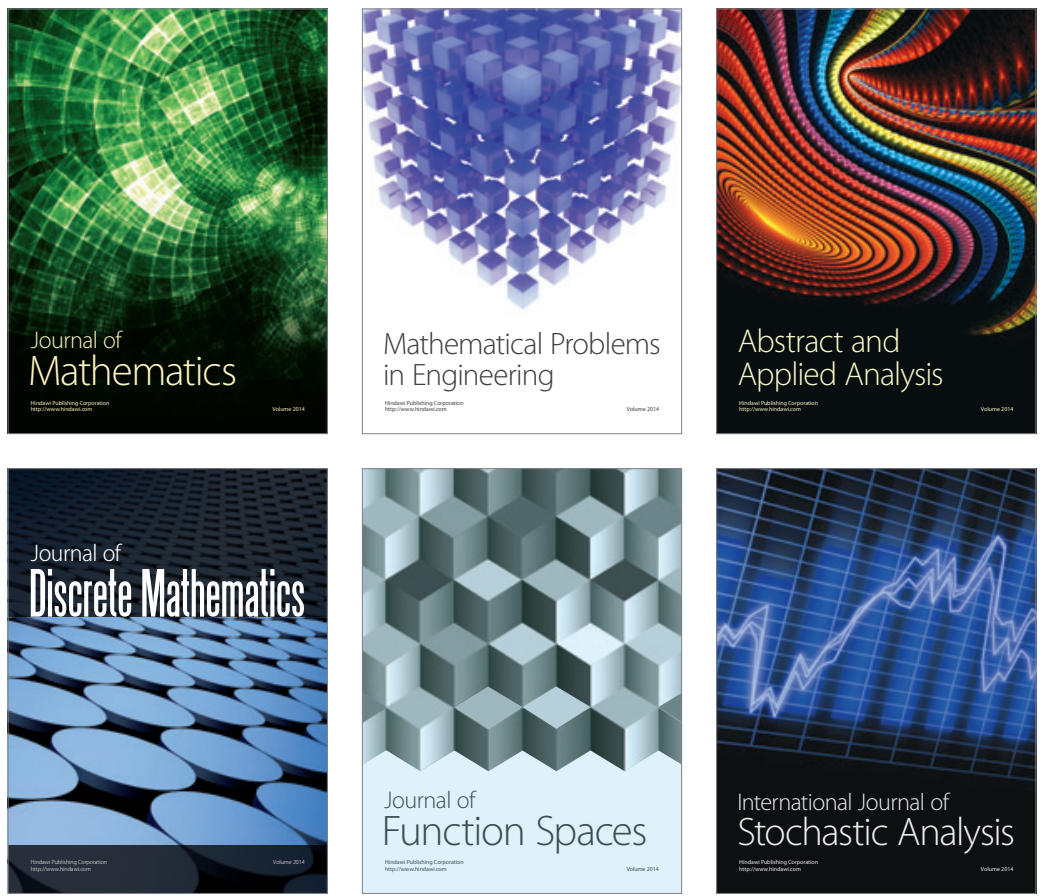

Journal of

Function Spaces

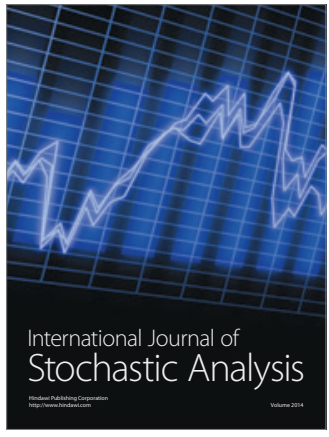

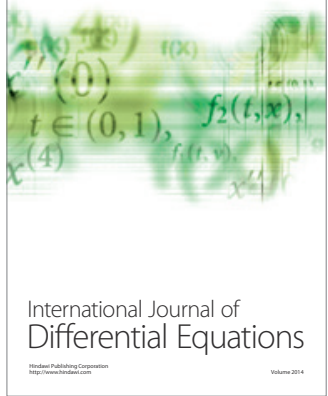
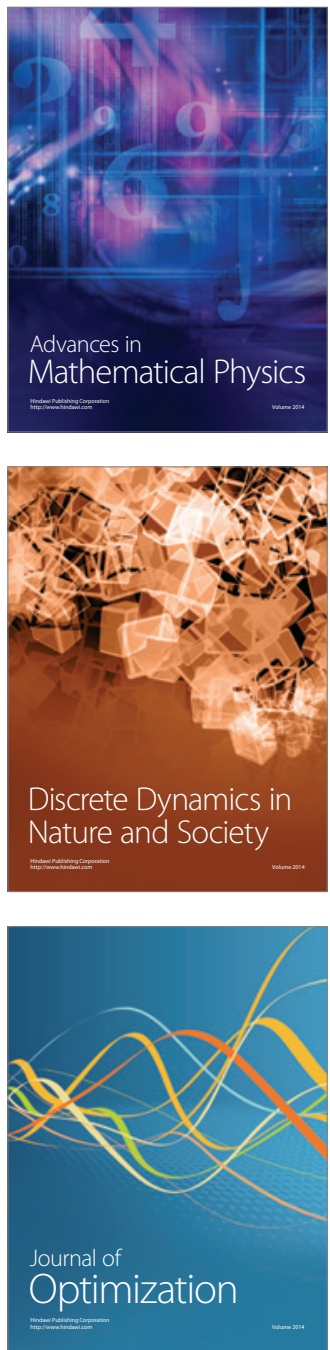